\title{
Electrical stimulation in obstructive sleep apnoea: the less invasive the better?
}

To the Editors:

We read with interest the article by EASTWOOD et al. [1] on bilateral hypoglossal nerve stimulation for treatment of adult obstructive sleep apnoea (OSA), the BLAST OSA trial. The authors present data on a novel approach, the Genio system, to stimulate the hypoglossal nerve and provide neuromuscular tone to the genioglossus, the main dilator muscle of the upper airway, to improve upper airway patency in OSA. The primary outcomes of the BLAST OSA trial focus on indices describing severity of OSA, the apnoeahypopnoea index (AHI), and the safety of this approach. The AHI improved by 10.8 events per hour at 6 months, leading to a symptomatic improvement, as measured by the Epworth Sleepiness Scale and the Functional Outcome of Sleep Questionnaire (FOSQ-10), and any serious adverse events observed were related to the surgical procedure; most minor adverse events wore off during the 6-month follow-up period.

Electrical stimulation for the treatment of OSA has been repeatedly tested since the 1980s, with more feasible approaches being developed invasively, as described in the STAR trial [2], as well as non-invasively, as explored in the TESLA trial [3]. While there are long-term follow-up data to underline the continued efficacy for the invasive methodology [4], "hybrid" technology [1, 5] and entirely non-invasive transcutaneous electrical nerve stimulation [6] are methods that are currently being tested and refined. The advantages of less and non-invasive technology, as pointed out by EASTWOOD et al. [1], are manifold and lie in the safety aspects and ease of use; the low risk profile and their cost efficiency will determine access to the technology and the eventual availability for many patients [7]. In addition, sleep medicine is moving towards new therapeutic concepts that may include combinations of different treatments in a single patient with OSA, and this favours easy-to-use methods.

Whilst EASTWOOD et al. [1] provide insights into many aspects of electrical stimulation in OSA, the study raises further points that could help to advance future research in this field, as follows.

First, electrical stimulation in OSA is moving from an entirely invasive to a less invasive approach, the "hybrid" solution provided by the Genio ${ }^{T M}$ system; to complete the approach, non-invasive electrical stimulation has already been developed [3] and is pending future trial results [6].

Secondly, EASTwOoD et al. [1] describe a previous trial that used the Apnex Medical device (NCT01446601), a pivotal trial that failed to show benefits due to the unexpected improvements observed in the control arm. While it remains difficult to design a surgical intervention with a control group that is adequately blinded, it is surprising that a study design was chosen for the BLAST OSA trial that contains a single arm only.

Thirdly, the current study describes "responders" to the new technology. While there is never any treatment that is $100 \%$ effective for everyone, it would have been helpful to provide more data to understand the responder group better. Was "response" dependent on factors other than the airway anatomy, as described in the screening process? To what extent did neck circumference, body mass index or gender contribute? These are potential factors that have previously been associated with "response" and helped to define in- and exclusion criteria for randomised controlled trials in the field [2, 3]. A larger sample size with a greater spread in characteristics such as body mass index might be needed to answer this question. However, defining criteria for a likely treatment response are essential for novel therapeutic methods in OSA that are less effective than continuous positive airway pressure therapy (CPAP).

@ERSpublications

Electrical stimulation for the treatment of OSA can be delivered invasively, using hybrid methods as well as non-invasively http://bit.ly/2ogLBFP

Cite this article as: Pengo M, Schwarz EI, Steier J. Electrical stimulation in obstructive sleep apnoea: the less invasive the better? Eur Respir J 2020; 55: 1902013 [https://doi.org/10.1183/13993003.02013-2019]. 
Lastly, the authors claim that bilateral and intermittent hypoglossal nerve stimulation was unique to their technology. This is correct in that there are no other invasive methods describing this approach. However, given the non-invasive nature of other studies describing the bilateral approach, and those using intermittent and pre-determined stimulation as well $[3,6,8]$, this section of the trial discussion would have benefitted from mentioning the additional context of transcutaneous electrical nerve stimulation being developed for OSA treatment.

We are grateful to the authors for highlighting the use of electrical stimulation in OSA. Patients with OSA who do not succeed in using CPAP in the long term require additional therapeutic alternatives. Non-invasive technologies using electrical stimulation are typically available for patients who would not necessarily qualify for more invasive procedures, be it due to physiological or anatomical factors, contraindications for the procedure or associated healthcare costs. We look forward to learning more about these and related methodologies in the near future.

Martino Pengo ${ }^{1}$, Esther Irene Schwarz ${ }^{2}$ and Joerg Steier $\oplus^{3}$

${ }^{1}$ Sleep Disorder Centre, IRCCS Istituto Auxologico Italiano, Milan, Italy. ${ }^{2}$ Dept of Pulmonology and Sleep Disorders Centre, University of Zurich, Zurich, Switzerland. ${ }^{3}$ Centre of Human and Applied Physiology (CHAPS), King's College London, London, UK.

Correspondence: Joerg Steier, Guy's and St Thomas' NHS Foundation Trust, Lane Fox Unit/Sleep Disorders Centre, South Wing, Ground Floor, Westminster Bridge Road, London, SE1 7EH, UK. E-mail: joerg.steier@gstt.nhs.uk

Received: 13 Oct 2019 | Accepted: 17 Oct 2019

Conflict of interest: M. Pengo has nothing to declare. E.I. Schwarz has nothing to declare. J. Steier is named inventor on a patent for Kings College London/Guys \& St Thomas' NHS Foundation Trust for an apparatus to treat snoring and sleep apnoea using electrical stimulation.

\section{References}

1 Eastwood PR, Barnes M, MacKay SG, et al. Bilateral hypoglossal nerve stimulation for treatment of adult obstructive sleep apnoea. Eur Respir J 2020; 55: 1901320.

2 Strollo P, Soose RJ, Maurer JT, et al. Upper-airway stimulation for obstructive sleep apnea. N Engl J Med 2014; 370: 139-149.

3 Pengo M, Xiao S, Ratneswaran C, et al. Randomised sham-controlled trial of transcutaneous electrical stimulation in obstructive sleep apnoea. Thorax 2016; 71: 923-931.

4 Costantino A, Rinaldi V, Moffa A, et al. Hypoglossal nerve stimulation long-term clinical outcomes: a systematic review and meta-analysis. Sleep Breath 2019; in press [https://doi.org/10.1007/s11325-019-01923-2].

5 Bisogni V, Pengo MF, De Vito A, et al. Electrical stimulation for the treatment of obstructive sleep apnoea: a review of the evidence. Expert Rev Respir Med 2017; 11: 711-720.

$6 \mathrm{He} \mathrm{N}$, Al-Sherif M, Nido M, et al. Domiciliary use of transcutaneous electrical stimulation for obstructive sleep apnoea: a conceptual framework for the TESLA home porgramme. J Thorac Dis 2019; 11: 2153-2164.

7 Pengo MF, Steier J. Emerging technology: electrical stimulation in obstructive sleep apnoea. J Thorac Dis 2015; 7: $1286-1297$.

8 Steier J, Seymour J, Rafferty GF, et al. Continuous transcutaneous electrical stimulation in obstructive sleep apnea: a feasibility study. Chest 2011; 140: 998-1007. 